\title{
The real seroprevalence of SARS-CoV-2 in France and its consequences for virus dynamics.
}

Chloe Dimeglio ( $\square$ dimeglio.c@chu-toulouse.fr)

Toulouse University Hospital Centre, Virology Laboratory

Jean-Michel Loubes

Toulouse Mathematics Institute

Marcel Miedougé

Toulouse University Hospital Centre, Virology Laboratory

\section{Fabrice Herin}

Toulouse University Hospital Centre, Occupational Diseases Department

Jean-Marc Soulat

Toulouse University Hospital Centre, Occupational Diseases Department Jacques Izopet

Toulouse University Hospital Centre, Virology Laboratory

\section{Method Article}

Keywords: SARS-CoV-2, seroprevalence, statistical model, serological tests, COVID-19

Posted Date: July 7th, 2020

DOl: https://doi.org/10.21203/rs.3.rs-40015/v1

License: (c) (i) This work is licensed under a Creative Commons Attribution 4.0 International License. Read Full License 
The real seroprevalence of SARS-CoV-2 in France and its consequences for virus dynamics.

Chloé Dimeglio ${ }^{1,2^{*}}$, Jean-Michel Loubes ${ }^{3}$, Marcel Miedougé2 ${ }^{2}$ Fabrice Herin ${ }^{4}$, Jean-Marc Soulat ${ }^{4}$, Jacques Izopet ${ }^{1,2}$

${ }^{1}$ UMR Inserm, U1043; UMR CNRS, U5282, Centre de Physiopathologie de Toulouse Purpan (CPTP), Toulouse 31300, France

${ }^{2} \mathrm{CHU}$ Toulouse, Hôpital Purpan, Virology Laboratory, 31300 France

${ }^{3}$ Université de Toulouse, Institut de Mathématiques de Toulouse, Toulouse 31400, France

${ }^{4}$ Occupational Diseases Department, Toulouse-Purpan University Hospital, Toulouse, 31000, France

*To whom correspondence should be addressed

\begin{abstract}
The SARS-CoV-2 virus has spread world-wide since December 2019, affecting millions of people and killing thousands. The recent use of serological tests to measure the effective seroprevalence of SARS-CoV- 2 in the general population has led us to assess the consequences of a much lower than expected seroprevalence on the real dynamics of the virus in the population.

Our prediction models assumed a $4.2 \%$ seroprevalence in Occitania and it has turned out to be only 1.3\% (Cl 95\%: 0.6\% - 2.6\%, binomial method). This implies a lower than expected virus transmission rate, which may be due to infectivity varying with the patient's symptoms or to a constraint due to an uneven population geographical distribution.
\end{abstract}

Keywords: SARS-CoV-2, seroprevalence, statistical model, serological tests, COVID-19

\title{
Introduction
}

The severe acute respiratory syndrome coronavirus 2 (SARS-CoV-2) that emerged in Wuhan, China in December 2019 has spread largely by sustained human-to-human transmission (1). The WHO declared the resulting disease a pandemic (2). The virus, which causes severe respiratory illness in susceptible individuals, has spread so rapidly that it has led most affected countries to lockdown their populations in order to break the dynamics of the virus's evolution. Many models have used the proportion of SARS-CoV-2 infected people tested by RT-PCR tests to predict the course of the epidemic $(3,4,5)$. Our statistical model for predicting the spread of SARS-CoV-2 in France is based on a diffusion and transmission coefficient that varies with an individual's age, the likelihood of contagion, and two administration parameters (lockdown and quarantine) (4). It indicated that the seroprevalence in France at the end of confinement would be no greater than 17.5\% (4). Recent results of serological tests measuring the effective seroprevalence of SARS-CoV- 2 in the general population have led us to assess the consequences of a much lower seroprevalence on the real virus dynamics in the population.

\section{Results}


We define $I_{N, R}$ as the real seroprevalence on day $N$ at the end of lockdown and $I_{N, E}$ as the seroprevalence estimated by the model on the same day.

We assumed that there were 3 untested SARS-CoV-2-contagious people in France when the first 3 cases were detected on January 24 ( $50 \%$ of asymptomatic cases). We assume that the number of tests carried out $(\alpha)$ has remain fixed, since the virology laboratories have never been saturated, and that the number of days a person is contagious $N_{c}$ is equal to 20 (6).

The quarantine constraint $q$ is considered to be negligible.

\section{a. Seroprevalence}

Lockdown began on March 17 in France and ended on May 11. The predicting model indicated that the seroprevalence in Occitania before March 17 was $0 \%$, and that it was $4.2 \%$ after lockdown. The serological test data obtained by the Toulouse Virology Laboratory show that the real seroprevalence in Occitania is $1.3 \%$ ( $\mathrm{Cl} 95 \%$ : $0.6 \%-2.6 \%$, binomial method). These data were obtained from a population of 613 individuals who were exposed to the virus to varying extents mimicking the general population in Occitania.

Consequently, it is quite possible that the national seroprevalence of SARS-CoV-2 is also lower than expected.

\section{b. Consequences for SARS-CoV-2 dynamics}

We have $I_{N, R}<I_{N, E}$

According to equation (3), $I_{N, R}=\left(I_{N-1}+P_{N-1}^{20}+Q_{N-1}^{20}\right)_{R}, I_{N-1, R}=\left(I_{N-2}+P_{N-2}^{20}+Q_{N-2}^{20}\right)_{R}$ and finally,

$$
I_{N, R}=\left(I_{1}+\sum_{n=1}^{N-1} P_{n}^{20}+\sum_{n=1}^{N-1} Q_{n}^{20}\right)_{R} \text { with } I_{1}=0 \text { (nobody immunized on day one) }
$$

(A) becomes $\left(\sum_{n=1}^{N-1} P_{n}^{20}+\sum_{n=1}^{N-1} Q_{n}^{20}\right)_{R}<\left(\sum_{n=1}^{N-1} P_{n}^{20}+\sum_{n=1}^{N-1} Q_{n}^{20}\right)_{E}$

Since $\forall n<20, P_{n}^{20}=0$ and $Q_{n}^{20}=0$, (A) becomes

$$
\left(\sum_{n=20}^{N-1} P_{n}^{20}+\sum_{n=20}^{N-1} Q_{n}^{20}\right)_{R}<\left(\sum_{n=20}^{N-1} P_{n}^{20}+\sum_{n=20}^{N-1} Q_{n}^{20}\right)_{E}
$$

According to equation (1), we have:

$$
\begin{aligned}
& P_{n}^{20}=P_{n-1}^{19}(1-\alpha) \\
& P_{n-1}^{19}=P_{n-2}^{18}(1-\alpha)
\end{aligned}
$$

Finally, $P_{n}^{20}=P_{n-19}^{1}(1-\alpha)^{19}$ with $P_{n-19}^{1}$ defined by equation (2).

Similarly, $Q_{n}^{20}$ is defined by equation (4) as:

$$
\begin{aligned}
& Q_{n}^{20}=Q_{n-1}^{19}+P_{n-1}^{19} \cdot \alpha \text { with } Q_{n-1}^{19}=Q_{n-2}^{18}+P_{n-2}^{18} \cdot \alpha \\
& Q_{n}^{20}=Q_{n-2}^{18}+P_{n-2}^{18} \cdot \alpha+P_{n-2}^{18} \cdot \alpha \cdot(1-\alpha)
\end{aligned}
$$


Finally, $Q_{n}^{20}=Q_{n-19}^{1}+P_{n-19}^{1} \cdot \sum_{m=0}^{18} \alpha \cdot(1-\alpha)^{m}$ with $Q_{n-19}^{1}=0$ (no quarantine on day one) and $P_{n-19}^{1}$ defined by equation (2).

(A) becomes:

$$
\begin{aligned}
\left(\sum_{n=20}^{N-1} \frac{S_{n}}{N t} \cdot\left[\sum_{i} P_{n-20}^{i} \cdot R_{0}^{i} \cdot c\right] \cdot\left[\sum_{m=0}^{18} \alpha \cdot(1-\alpha)^{m}+(1-\alpha)^{19}\right]\right) R \\
<\left(\sum_{n=20}^{N-1} \frac{S_{n}}{N t} \cdot\left[\sum_{i} P_{n-20}^{i} \cdot R_{0}^{i} \cdot c\right] \cdot\left[\sum_{m=0}^{18} \alpha \cdot(1-\alpha)^{m}+(1-\alpha)^{19}\right]\right) E
\end{aligned}
$$

Which equals:

$$
\left(\sum_{n=20}^{N-1} \frac{S_{n}}{N t} \cdot\left[\sum_{i} P_{n-20}^{i} \cdot R_{0}^{i} \cdot c\right]\right)_{R}<\left(\sum_{n=20}^{N-1} \frac{S_{n}}{N t} \cdot\left[\sum_{i} P_{n-20}^{i} \cdot R_{0}^{i} \cdot c\right]\right)_{E}
$$

The three possible explanations for why the real seroprevalence is lower than expected are:

1. $c_{R}<c_{E}$

2. $P_{n, R}<P_{n, E}$

3. $\left(R_{0}^{i}\right)_{R}<\left(R_{0}^{i}\right)_{E}$

\section{Discussion}

We used a discretized SIR model to predict the dynamics of SARS-CoV-2 infections that takes into account various factors involved in the virus dynamics. Lockdown began on 17 March in France, and was gradually relaxed from May 11 . Under these circumstances, the seroprevalence at the end of lockdown was estimated to be $17.5 \%$ in France and $4.2 \%$ in Occitania (4). But the serological tests carried out since the end of May indicate that the real seroprevalence is lower, at 1.3\% (Cl 95\% : $0.6 \%-2.6 \%$, binomial method) in Occitania, which suggests that the national seroprevalence is also lower. This is consistent with the findings of a study indicating that $4.4 \%$ (range $2.8 \%$ to $7.2 \%$ ) of the French population (2.8 million people) had been infected (7).

This could be because the actual lockdown constraint $c_{R}$ was more stringent than expected $c_{E}$. This seems unlikely since we have set the lockdown constraint at $80 \%$ and that it was neither strictly respected nor as restrictive as expected, given that some people continued to go out to work.

The second possibility is that the fraction of the population initially reached but not detected $P_{n, R}$ was smaller than expected, $P_{n, E}$, or that the asymptomatic people were less contagious than those who were symptomatic. The Center for Disease Control and Prevention (CDC) has reported that researchers in Singapore concluded that asymptomatic people were the source of $44 \%$ of diagnosed COVID-19 cases (8). Two studies published in late May indicated that many COVID-19-infected people could be asymptomatic. Another recent study reported that 104/128 (81 percent) of people on a cruise ship who tested positive for SARS-CoV-2 were asymptomatic (9). And yet another study found that $42 \%$ of those who tested positive for SARS-CoV-2 were without symptoms (10). The proportion of asymptomatic cases in our model was close to $50 \%$ but it is possible that the actual proportion was lower even if the assumed proportion remains within the possible range. The expected proportion of asymptomatic people could be higher than that actually observed if these asymptomatic people were less contagious than expected. Our model assumes that the transmission rate can vary with the day of infection but is independent of the patient's symptoms. However, the proportion of 
asymptomatic infected people could also be lower than expected if these people were less contagious. This relationship was favored in a recent study looking at the symptoms of 455 cases who were in contact with a confirmed but asymptomatic SARS-CoV-2 patient (11).

Our last explanation for a lower than expected seroprevalence assumes that the transmission rate was lower than expected: $\left(R_{0}^{i}\right)_{R}<\left(R_{0}^{i}\right)_{E}$. The WHO set this rate at an average of 2.2 (8), with occasional large variations. A recent review comparing 12 studies that estimated the $R_{0}$ for COVID19 found values of from 1.5 to 6.68 (14). The 2.2 average rate may be less important in reality or it may vary according to an individual's symptoms, as previously discussed. It is also possible that the rate itself does not vary but that its constraints do. Our model assumes that the virus is uniformly distributed throughout the population, but the first episodes of virus infection occurred in clusters, which assumes concentrations of population in given places without necessarily any communication between the clusters. A recent analysis of the haplotypes of SARS-CoV-2 infections in Iceland concluded that the geographical distribution of clades was highly structured (15). Thus the rate of virus transmission may be that set by the WHO within clusters but it could vary outside them or be much lower because of the uneven population density.

Serological testing has shown that the seroprevalence of SARS-CoV-2 is much lower than expected. In particular our prediction models assumed a $4.2 \%$ seroprevalence in Occitania and it turned out to be only $1.3 \%$. This implies slower than expected virus transmission, perhaps due to variations in infectivity influenced by a patient's symptoms or to geographical constraints on the population distribution. The lack of protective immunity after reduction of lockdown justify massive testing for SARS-CoV-2, tracking contacts and isolation to contain the epidemic.

\section{Methods}

\section{Serological tests}

Total plasma anti-SARS-CoV-2 antibodies ( $A b)$ were detected using an enzyme linked immunosorbent assay (ELISA) kit (Beijing Wantai Biological Pharmacy Entreprise Co., Ltd, China) according to the manufacturer's instructions. This assay is based on a recombinant antigen containing the SARS-CoV-2 spike protein receptor-binding domain (RBD). The sensitivity using samples collected 15 to 45 days post symptom-onset or after contact with a SARS-CoV-2 case, including asymptomatic patients, was $100 \%$ (CI 95\% : $88.2 \%-100 \%$ ) and the specificity using plasma collected before the outbreak of SARSCoV-2 was $100 \%$ (Cl 95\% : 82.1\% - 100\%) (14).

\section{Statistical model}

We considered the variables $\left(S_{n}, P_{n}, Q_{n}, I_{n}\right)$.

$S_{n}$ is the number of healthy people on day $n, P_{n}^{i}$ is the number of undetected contagious carriers infected for $i$ days $\left(1 \leq i \leq N_{c}\right)$. Similarly, $Q_{n}^{i}$ is the number of detected contagious carriers infected for $i$ days $\left(1 \leq i \leq N_{c}\right.$ ) on day $n$, and $I_{n}$ is the number of people who were immunized.

$N_{c}$ is the number of days a person is contagious and $\alpha$ is the percentage of the population tested on each day. $R_{0}$ is the number of healthy people who a contagious person contacts and infects. We assume that the contagion coefficient varies over time and peaks when the virus load is maximal: 7 days after the start of infection (6). $N t$ is the total population at the start of the epidemic phase, $c$ is the multiplier for the pace of the epidemic throughout lockdown $(0 \leq c \leq 1)$, and $q$ is the same multiplier during the quarantine period $(0 \leq q \leq 1) . c$ and $q$ are set at 1 when there is no lockdown or quarantine. 
$N t$ is given by:

$N t=S_{n}+P_{n}+Q_{n}+I_{n}$

On transition from day $n$ to day $n+1$, we have:

$\forall 1 \leq i \leq N_{c}-1, P_{n+1}^{i+1}=P_{n}^{i}(1-\alpha)$

$P_{n+1}^{1}=\frac{s_{n}}{N t} \cdot\left[\sum_{i} P_{n}^{i} \cdot R_{0}^{i} \cdot c+\sum_{i} Q_{n}^{i} \cdot R_{0}^{i} \cdot q\right]$

$I_{n+1}=I_{n}+P_{n}^{N_{c}}+Q_{n}^{N_{c}}$

$Q_{n+1}^{1}=0$ (no quarantine on day one, test results needed)

$\forall 1 \leq i \leq N_{c}-1, Q_{n+1}^{i+1}=Q_{n}^{i}+P_{n}^{i} \cdot \alpha$

The model is a discretized version of a Susceptible Infectious and Recovered (SIR)-type model (15)

We set $R_{0}=2.2(16)$ at its peak.

\section{CONFLICT OF INTEREST}

The authors have no conflict of interest to declare.

\section{ACKNOWLEDGEMENTS}

The English text was edited by Dr Owen Parkes.

\section{References}

1. Perlman, S. Another Decade, Another Coronavirus N. Engl. J. Med. https://doi.org/10.1056/NEJMe2001126 (2020).

2. WHO Virtual press conference on COVID-19-11 March (2020). https://www.who.int/docs/default-source/coronaviruse/transcripts/who-audioemergencies-coronavirus-press-conference-full-and-final11 mar2020.pdf? sfvrsn=cb432bb3 2 (2020).

3. Kissler, S.M, Tedijanto, C., Goldstein, E., Grad, Y.H and Lipsitch, M. Projecting the transmission dynamics of SARS-CoV-2 through the postpandemic period Science : 860-868 (2020).

4. Dimeglio, C. et al. The SARS-CoV-2 seroprevalence is the key factor for deconfinement in France. Journal of Infection ISSN: 0163-4453 (2020).

5. Wu, J.T., \& Leung, K. Nowcasting and forecasting the potential domestic and international spread of the 2019-ncov outbreak originating in wuhan, china: a modelling study. Lancet 395(3) (2020).

6. Chang, M.G et al. Time Kinetics of Viral Clearance and Resolution of Symptoms in Novel Coronavirus Infection. Am.J.Res.Crit.Care Med. (2020). 
7. Salje $\mathrm{H}$, et al. Estimating the burden of SARS-CoV-2 in France. Science (2020)

8. He, X., et al. Temporal dynamics in viral shedding and transmissibility of COVID-19. Nat Med 26, 672-675 (2020).

9. Ing AJ, Cocks C, Green JP COVID-19: in the footsteps of Ernest ShackletonThorax Published Online First: 27 May 2020. doi: 10.1136/thoraxjnl-2020-215091

10. Yang R, Gui X, Xiong Y. Comparison of Clinical Characteristics of Patients with Asymptomatic vs Symptomatic Coronavirus Disease 2019 in Wuhan, China. JAMA Netw Open;3(5) (2020).

11. Gao,M., et al. A study on infectivity of asymptomatic SARS-CoV-2 carriers, Respiratory Medicine, 169 (2020).

12. Liu Y, Gayle AA, Wilder-Smith A, Rocklöv J. The reproductive number of COVID-19 is higher compared to SARS coronavirus. J Travel Med (2020).

13. Gudbjartsson DF, et al. Spread of 297 SARS-CoV-2 in the Icelandic Population. N Engl J Med. (2020).

14. Abravanel F. et al. Clinical performance of a rapid test compared to a microplate test to detect total anti SARS-CoV-2 antibodies directed to the spike protein. Journal of Clinical Virology In press (2020).

15. Kermack, W. O. and McKendrick, A. G. "A Contribution to the Mathematical Theory of Epidemics." Proc. Roy. Soc. Lond. A 115, 700-721, (1927)

16. WHO Report of the WHO-China Joint Mission on Coronavirus Disease.(COVID-19). Available at:

https://www.who.int/docs/default-source/coronaviruse/who-china-joint-mission-on-covid19-final-report.pdf (2019). 